\title{
Circ_001209 aggravates diabetic retinal vascular dysfunction through regulating miR-15b-5p/COL12A1
}

Fang Wang ${ }^{1,2}$ and Meixia Zhang ${ }^{1 *}$

\begin{abstract}
Objective: Diabetic retinopathy, a common complication of diabetes mellitus and a major cause of blindness. circRNAs spongs target miRNA and thus influencing mRNA expression in DR. We investigated the mechanism of circ_001209 in regulating diabetic retinal vascular dysfunction.

Methods: QRT-PCR analysis was performed to detect the expression of miR-15b-5p, COL12A1 and circ_001209 in human retinal vascular endothelial cells (HRVECs) under high glucose conditions. Western blot assay, wound healing assay, transwell assay and tube formation were used to explore the roles of circ_001209/miR-15b-5p/COL12A1 in retinal vascular dysfunction. Bioinformatics analysis and luciferase reporter, RNA-FISH, and overexpression assays were performed to reveal the mechanisms of the circ_001209/miR-15b-5p/COL12A1 interaction. TUNEL staining and H\&E staining were used to evaluate the pathological changes in streptozotocin (STZ)-induced DR in rats.

Results: Downregulation of miR-15b-5p under HG conditions promoted proliferation, migration, and tube formation of HRVECS. QRT-PCR and western blot results revealed that miR-15b-5p affected the HRVECs function through targeting COL12A1. Under HG conditions, circ_001209, which acts as a sponge of miR-15b-5p, is upregulated. Besides, overexpression of circ_001209 can affect HRVEC function and aggravate retinal injury in diabetic rats.
\end{abstract}

Conclusion: Upregulation of circ_001209 contributes to vascular dysfunction in diabetic retinas through regulating miR-15b-5p and COL12A1, providing a potential treatment strategy for diabetic retinopathy.

Keywords: circ_001209, Diabetic retinal, HRVECs, miR-15b-5p, COL12A1

\section{Introduction}

Diabetic retinopathy (DR), as one of the most common complications of diabetes mellitus (DM), which is the leading cause of vision impairment and even blindness worldwide [1]. The disease reportedly affects more than 100 million patients worldwide as of 2010 and will continue to rise, reaching an estimated 190 million by 2030 [2]. Previous studies [3] have reported that diabetic retinas are mainly accompanied by microaneurysms,

*Correspondence: coretina@gmail.com

1 Department of Ophthalmology, West China Hospital, Sichuan University, Chengdu 610041, Sichuan, China

Full list of author information is available at the end of the article punctate hemorrhage, cotton-floss plaques and retinal microvascular abnormalities under continuous hyperglycemia stimulation. The pathological manifestation of diabetic retinopathy is that the patient's continuous hyperglycemia environment leads to the apoptosis of retinal peripheral vascular cells, promotes the abnormal proliferation of retinal vascular endothelial cells, and seriously affects the function of retinal blood vessels [46]. At present, the primary treatment strategy [7] is still to control the body's blood glucose concentration and alleviate the sustained damage caused by hyperglycemia stimulation, but the mechanism of diabetic retinopathy targeting vascular endothelial function is still unclear. Thereby, further research on the mechanism of DR 
progression is urgently needed to provide a new effective therapeutic strategy for DR.

MicroRNAs (miRNAs) are small, non-coding RNAs that interact with the 3' untranslated region (UTR) of mRNAs to directly regulate mRNA degradation and translation, and regulates gene expression. It has been reported that miRNAs have a wide range of regulatory functions, including cell proliferation, differentiation, apoptosis, autophagy and other physiological and pathological processes, which may indirectly affect physiological functions and pathological processes of the body [8]. Increasing evidences [9] have showed that miRNAs are associated with the pathological process of DR. Xue et al. [10] found that miR-200-3p plays a protective role in inhibiting the proliferation of diabetic retinopathy cells by blocking the TGF-2/Smad pathway. Ji et al. [11] suggested that miR-34a promotes apoptosis of RVECs by targeting SIRT1 in DR rats, which provide a valuable target for DR treatment. In Ye et al. [12] reported, miR-15b plays a protective role by regulating the TNF- $\alpha /$ SOCS3 signaling pathway against hyperglycemia-induced retinal endothelial cell apoptosis.

CircRNA, as a novel star in the study of ncRNA, has a single-chain covalent closed structure, excluding 50 caps and 30 multi-A-tails, and its mechanism of action in the field of biomedicine has attracted wide attention [13]. With the rapid development of biological technologies, circRNAs have been found to interact with RNA-binng proteins (RBPs) to regulate RNA splicing or transcription and translation into peptides or proteins to regulate various biological processes, including cell differentiation, proliferation, apoptosis, inflammation and invasion, in addition to acting as sponges for microRNAs [14]. It is reported that circ_0005015 may serve as a novel potential biomarker of DR, which could facilitate retinal endothelial angiogenic function and aggravate the progression of DR. Combined with the database to predict the upstream circRNA of miR-15b-5p, we further explored the regulatory mechanism of circ_001209 in diabetic retinopathy.

In our study, we analyzed the expression of miR-15b-5p and circ_001209 in the HRVECs induced by HG and diabetic rats. We found that overexpression of circ_001209 could promote the cell proliferation, migration and vascularization and in HG-induced HRVECs. Besides, we also confirmed that circ_001209 could aggravate the progression of $\mathrm{DR}$ in vivo through interaction with miR-15b-5p/COL12A1.

\section{Materials and methods}

\section{Cell culture and transfection}

Human retinal vascular endothelial cells (HRVECs) were purchased from Cell Systems Corporation (UK). HRVECs were cultured in a basic endothelial cell culture medium
(5 mM glucose) containing 10\% FBS (fetal bovine serum) in an incubator at $37{ }^{\circ} \mathrm{C}$ with $5 \% \mathrm{CO}_{2}$, called the control group. In the HG group, the cells were treated with $25 \mathrm{mM}$ glucose (Solarbio, Beijing). When the HRVECs coverage reached $80 \%$ of the culture flask, cell passage was started, and cells at passage three to eight were selected for subsequent experiments. HRVECs were transfected with miR-15b-5p mimics, mimics control, pcDNA3.1 (empty plasmid) and pcDNA3.1-COL12A1 (RiboBio, China) by Lipofectamine 3000 (Thermo Fisher Scientific, USA) according to the manufacturer's instructions. The relative expressions of miR-15b-5p and COL12A1 were detected by qRT-PCR analysis at $48 \mathrm{~h}$ after transfection.

\section{Diabetic retinopathy model}

The study was approved by the Animal Ethics Committee of West China Hospital, and all experiments are conducted in accordance with guidelines for the use and care of laboratory animals, following the principles of reducing animal numbers and suffering. A total of 80 SpragueDawley male rats $(160 \pm 10 \mathrm{~g})$ were purchased from Beijing huafukang bioscience co., LTD. Rats were kept in ventilated cages in controlled room (12 h light-dark cycle) with a temperature of $25 \pm 1{ }^{\circ} \mathrm{C}$ and humidity of $50 \%$. Under the conditions of the environment permits, the rats were allowed to free access to water and food. After seven days of adaptive feeding, animal experiments were started. Diabetic retinopathy rats were induced by intraperitoneal injection of $65 \mathrm{mg} / \mathrm{kg} \mathrm{STZ}$ (streptozotocin, Sigma, USA) [15]. The rats were fasted for $12 \mathrm{~h}$ before injection. Then, STZ was dissolved in $10 \mathrm{mM}$ citrate buffer $(\mathrm{pH}=4.5)$ and injected continuously for 7 days to induce DR model. At the same time, the sham rats were intraperitoneally injected with sodium citrate buffer. After the last injection, blood was collected from the tail vein for blood glucose detection. A blood glucose meter was used to detect blood glucose concentration > $16.7 \mathrm{mmol} / \mathrm{L}$ for consecutive seven days, indicating that the diabetes model was successful. Besides, fasting blood glucose was monitored and recorded every other week after the end of injection. Adequate food and water were given during the feeding period and keep the cage clean every day.

\section{Intravitreal injection of AAV-circ_001209}

The right eye of the rats was given topical treatments with $1 \%$ atropine sulfate and $2.5 \%$ phenylepinephrine hydrochloride to expand the eyes, making the injection more convenient. The human circ_001209 sequence was cloned into AAV2 vector and packaged into adenoassociated virus (AAVs) by General Biotech. Four $\mu \mathrm{L}$ AAV-circ_001209 or AAV-ciR-NC (empty vector) 
$\left(7 \times 10^{13} \mathrm{vg} / \mathrm{mL}\right)$ was injected into the vitreous cavity of rats using a microinjector. The relative expression level of circ_001209 in the retinal tissue was detected by qRTPCR analysis to evaluate the transfection efficiency.

\section{Quantitative real-time polymerase chain reaction (qRT-PCR) analysis}

Total RNA was extracted from retinal tissue and HRVECs using the TRIzol reagent (Invitrogen, USA) according to the protocols. The RNA was reverse-transcribed into cDNA using the PrimeScriptTM RT Reagent Kit (TaKaRa, Japan). SYBR Premix Ex TaqTM was used for amplification. GAPDH was used as an internal control for mRNA and circRNA, while U6 was selected as an internal control for miRNAs. The relative mRNA expressions in HRVECs and tissues were calculated using $2^{-\Delta \Delta \mathrm{Ct}}$ method.

\section{Western blotting assay}

Western blotting assay was used to detect the protein expression of COL12A1 and GAPDH in HRVECs. Firstly, proteins were extracted from the cells using RIPA lysis buffer. Protein concentration in the samples was determined by BCA kit and diluted to $10 \mu \mathrm{g} / \mu \mathrm{L}$ with PBS. Then, the samples were separated by $10 \%$ sodium dodecyl sulfate polyacrylamide gel electrophoresis and transferred to PVDF membrane (Millipore, USA). The membrane was blocked with $5 \%$ skim milk at room temperature for $3 \mathrm{~h}$, and the primary antibody (COL12A1 and GAPDH) was incubated overnight at $4{ }^{\circ} \mathrm{C}$. After washing the membrane for three times with TBST, the secondary antibody was incubated at $37^{\circ} \mathrm{C}$ for $1 \mathrm{~h}$. ECLchemiluminescent kit (Thermo Scientific, USA) was used to develop and quantify protein expression levels. Finally Image J was used to analyze the gray values of the protein bands, and GAPDH was used as the loading control.

\section{Wound healing assay}

Wound healing assay was used to detect the migration of HRVECs. As previously reported, the HRVECs were seeded in 24-well plate, and the cell confluency reached over $90 \%$, the scratch was made by a pipette tip. Then the cells were cultured for $24 \mathrm{~h}$, and the scratches were observed with a microscope (Leica, Germany) to evaluate the cell migration ability.

\section{Transwell assay}

Transwell assay was used to detect the cell migration and invasion. Briefly [16], the cells were seeded on the top layer of transwell chambers (Corning, USA) in serumfree medium, and then allowed to migrate to the lower compartment contained complete media with $10 \%$ FBS in an incubator at $37{ }^{\circ} \mathrm{C}$ for $24 \mathrm{~h}$. The migrated cells were fixed with fixative solution for $10 \mathrm{~min}$ and stained with $0.1 \%$ crystal violet for $15 \mathrm{~min}$. After washing with PBS, the cells were photographed and quantified with Image J software.

\section{Tube formation assay}

To investigate the ability of cell angiogenesis, tube formation assay was performed in vitro [17]. Growth Factor Reduced Matrigel matrix was evenly dispersed in 24-well plates and incubated at $37^{\circ} \mathrm{C}$ for $1 \mathrm{~h}$. The cells were seeded on the matrix surface at a density of $1 \times 10^{5}$ cells/well and cultured in an incubator for $24 \mathrm{~h}$. Finally, the capillary-like structures were observed under Nikon inverted microscope and its branches were analyzed by image J software.

\section{Dual luciferase reporter assay}

According to the database prediction, we designed primer sequences, and vectors containing relevant primer sequences were synthesized by general biosynthesis for subsequent experiments. 293T cells were seeded in 24-well plates $\left(1 \times 10^{5}\right.$ cell/well $)$ and co-transfected with circ_001209/COL12A1 wt or mut plasmid and miR15b-5p mimics or mimics control using Lipofectamine 3000 (Thermo Fisher Scientific, USA). At $48 \mathrm{~h}$ after transfection, luciferase activity was measured by the dual luciferase reporter assay system (Promega) following to the protocol [18].

\section{RNA fluorescence in situ hybridization (FISH)}

RNA FISH assay was detected using fluorescent In Situ Hybridization kit (RiboBio, China) following the manufacturer's protocol. The specific probes targeting circ_001209 and miR-15b-5p were used in the FISH experiment. In brief [19], HRVECs were fixed in $4 \%$ polyformaldehyde at room temperature for $15 \mathrm{~min}$ and washed twice with PBS. Subsequently, the penetration treatment was performed with $0.5 \%$ Triton. Then cells were hybridized overnight at $37{ }^{\circ} \mathrm{C}$ in darkness and humidity. The next day, the cells were washed with sodium citrate buffer and incubated in the blocking solution for $1 \mathrm{~h}$ at room temperature. The HRVECs were incubated with HRP-conjugated anti-biotin antibody at $4{ }^{\circ} \mathrm{C}$ overnight. Finally, images were observed and taken using a confocal microscope (Leica, Germany).

\section{Terminal deoxynucleotidyl transferase (TdT)-mediated dUTP biotin nick end labeling (TUNEL) staining}

Briefly, the retina tissues were embedded in paraffin embedded were made into $5 \mu \mathrm{m}$-thick sections, extended in warm water at $42{ }^{\circ} \mathrm{C}$, mounted, and baked. Subsequently, the resulting sections were added drop wise with $\mathrm{TdT}$ reaction solution and reacted in the dark for 
$1 \mathrm{~h}$. Then, the reaction was terminated through incubation with deionized water in drops for $15 \mathrm{~min}$. After the activity of endogenous peroxidase was blocked by hydrogen peroxide, the sections were added drop wise with working solution. Following reaction for $1 \mathrm{~h}$, the sections were rinsed, added with DAB solution in drops for color development, being counter-stained with hematoxylin for 20-30 s, and washed in running water again. Finally, they were dehydrated in increasing concentrations of alcohol sealed and observed. The apoptotic index was determined by counting the percentage of positive cells in 6 randomly chosen fields.

\section{Hematoxylin-eosin (H\&E) staining}

Retinal tissue was carefully separated from rats, gently washed with 1\% PBS (phosphate buffered saline), and fixed with $4 \%$ paraformaldehyde for $2 \mathrm{~h}$ for subsequent experiments. The tissue was embedded in paraffin and cut uniformly into $5-\mu \mathrm{m}$ thick sections. As described previously [15], sections were used for $H \& E$ staining to observe pathological changes in DR rats. After the staining, it was naturally air-dried, observed under a light microscope and photographed.

\section{Statistical analysis}

The in vitro experiments in the study were performed at least three independent replicates and the data was presented as mean \pm SEM. SPSS 22.0 software was used to analyze the data. The differences between two groups were analyzed by unpaired Student's $t$ test. Multiple comparisons were calculated using one-way analysis of variance (ANOVA), followed by Bonferroni multiple comparison test. $\mathrm{P}<0.05$ was considered statistically significant.

\section{Results}

MiR-15b-5p is low expressed in STZ-induced diabetic retinopathy tissues and affects function of HRVECs under high-glucose (HG) conditions.

We detected the expression levels of miR-15b-5p in STZ-induced diabetic retinopathy tissues and HRVECs induced by HG. As shown in Fig. 1A, compared with sham group, the expressions of miR-15b-5p was reduced significantly in STZ-induced diabetic retinopathy tissue. We cultured the HRVECs in high-glucose (HG) $(25 \mathrm{mM})$ and control group $(5 \mathrm{mM})$ and followed by qRTPCR analysis. The results (Fig. 1B) showed that compared with control group, the expression of miR-15b-5p was down-regulated markedly in HG group. To further investigate the role of miR-15b-5p in diabetic retinopathy, HRVECs were transfected with mimics control and miR-15b-5p mimics and cultured in HG and control conditions, respectively. The expression of miR-15b-5p was measured by qRT-PCR analysis in HRVECs in different groups. The results (Fig. 1C) revealed that compared with HG + mimics control group, the expression of miR15b-5p was increased significantly in HG + miR-15b-5p mimics group. Furthermore, we detected the invasion, migration, and angiogenesis of HRVECs induced by HG through wound healing assay, transwell assay and tube formation experiment. As shown in Fig. 1D-F, it showed that HG significantly increased the invasion, migration and tubular formation of HRVECs cells. Compared with HG + mimics control group, the effects of HG were reversed with $\mathrm{miR}+15 \mathrm{~b}-5 \mathrm{p}$ mimics. Taken together, we found that miR-15b-5p could significantly inhibit invasion, migration and tubular formation induced by HG.

\section{MiR-15b-5p regulates HRVEC function through targeting COL12A1}

Further experiments were performed to explore the effects of miR-15b-5p on HRVECs cell functions, such as cell invasion, migration and tube formation. First, according to Target Scan, Star base and microRNA.org, we predict the potential target genes of miR-15b-5p through bioinformatics analysis (Fig. 2A). QRT-PCR analysis was used to detect the difference of selected target genes. The results (Fig. 2B) showed that COL12A1 is the most significantly upregulated gene in HG-induced HRVECs. We further investigated whether the effects of miR-15b-5p on HRVECs were exerted through targeting COL12A1. Furthermore, TargetScan prediction and luciferase activity assay verified that miR-15b-5p had a binding relationship with COL12A1 (Fig. 2C). Luciferase activity assay results showed that a significant decrease in luciferase activity was observed in the group with the wild-type COL12A1 reporter (Luc-COL12A1-WT) plus miR-15b-5p mimics (Fig. 2D). In addition, we detected the expressions of COL12A1 in HG-induced HRVECs through qRTPCR assay and western blot. As shown in Fig. 2E, F, we found that compared with $\mathrm{HG}+$ mimics control group, the mRNA and protein expressions of COL12A1 in HG + miR-15b-5p mimics group was reduced significantly. The expressions of COL12A1 in HG+miR15b-5p mimics + pcDNA3.1-COL12A1 group was increased significantly compared with HG + miR-15b-5p mimics + pcDNA3.1 group. Moreover we evaluated the effects of miR-15b-5p in the HRVECs functions through targeting COL12A1. The wound healing results, transwell assay results and tube formation results (Fig. 2G-I) showed that compared with HG + mimics control group, the invasion, migration and tube formation of HRVECs was inhibited in HG + miR-15b-5p mimics group significantly. While the invasion, migration and tube formation of HRVECs in HG + miR-15b-5p mimics + pcDNA3.1COL12A1 group was enhanced significantly compared 


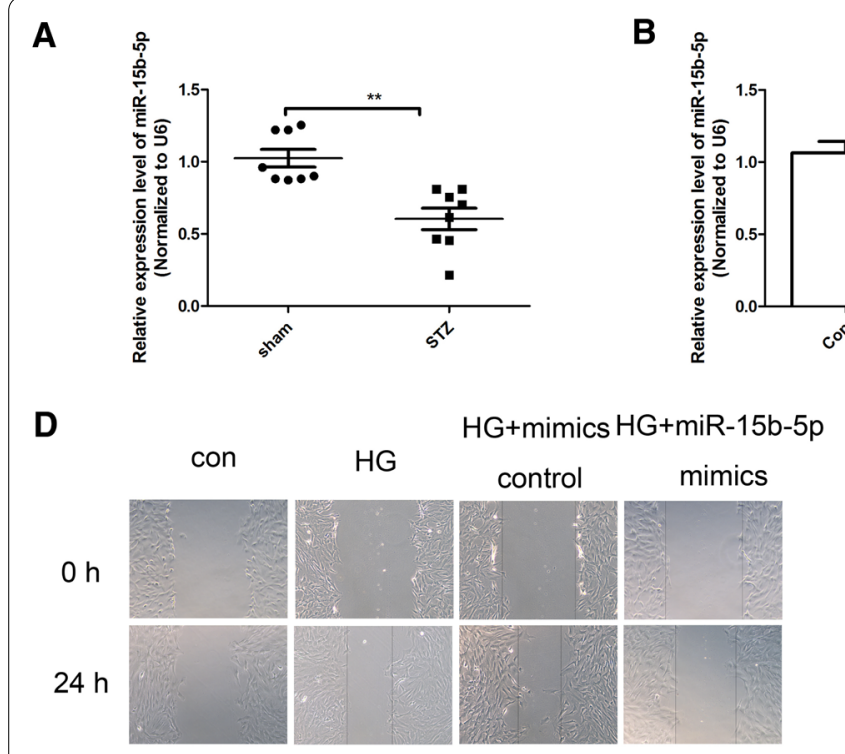

E
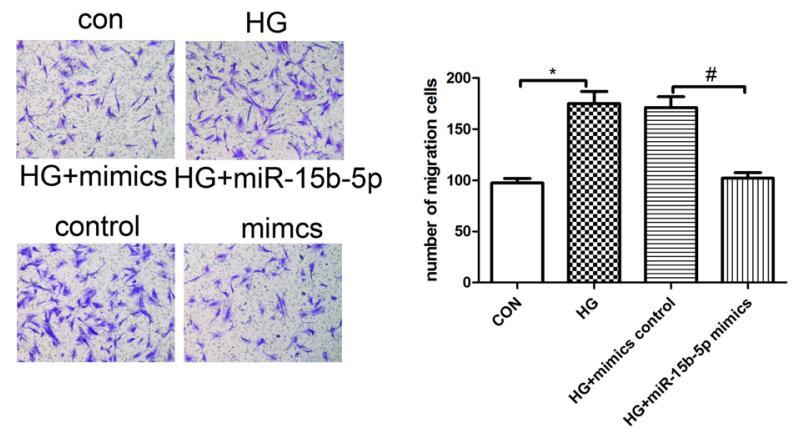

$\mathbf{F}$
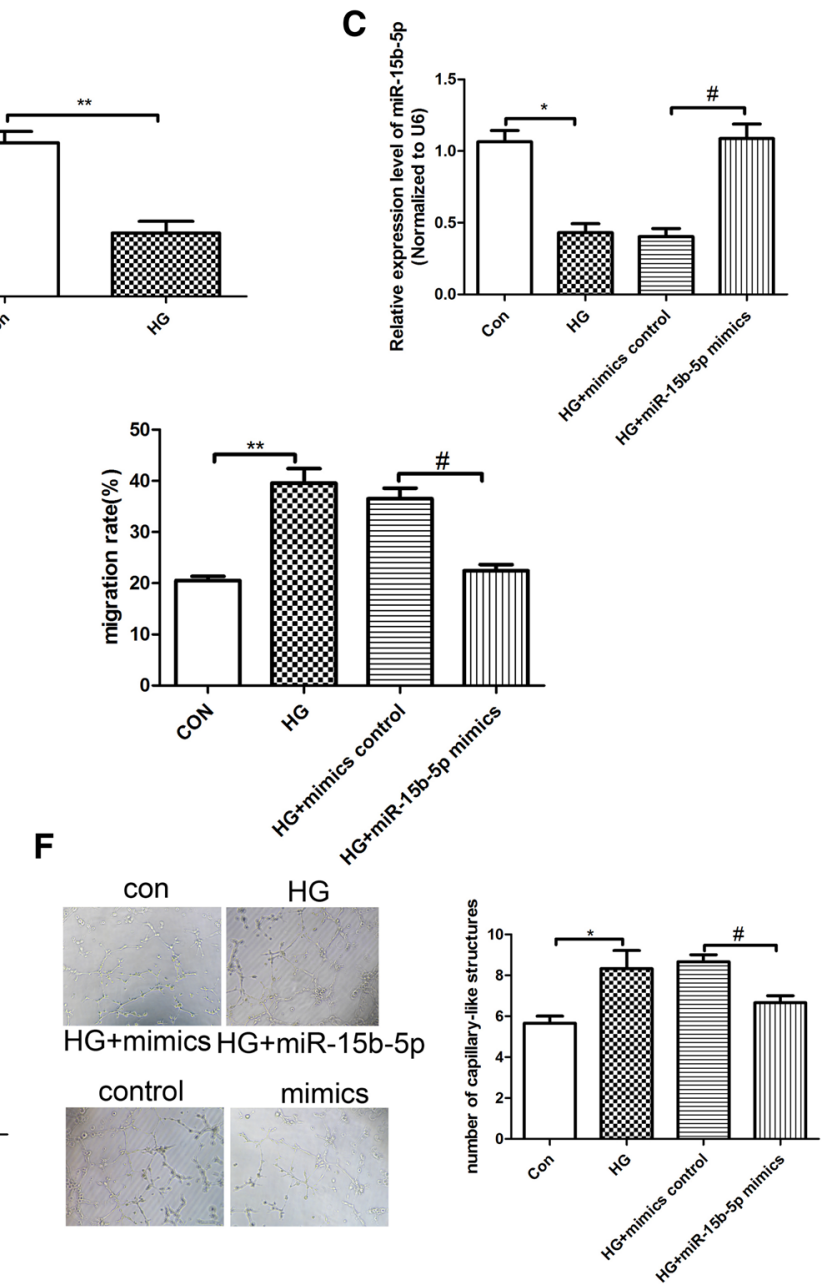

Fig. 1 MiR-15b-5p is low expressed in STZ-induced diabetic retinopathy tissues and affects function of HRVECs under high-glucose (HG) conditions. A The relative expression levels of miR-15b-5p, detected by qRT-PCR analysis, in sham group and diabetic rat retinas. B The qRT-PCR analysis was used to detect the expression level of miR-15b-5p in HRVECs induced by HG ( $25 \mathrm{mM}$ glucose). C The relative expression of miR-15b-5p in HRVECs was detected by qRT-PCR analysis. D Wounding healing assay was performed to detect the invasion ability in different groups. E Transwell assay was used to measure the migration ability in different groups. $\mathbf{F}$ The tubule-forming ability of cells was measured by the tubule formation experiment. The data were presented as mean $\pm \mathrm{SEM}, \mathrm{n}=3$ each group, vs con/sham group, ${ }^{*} \mathrm{P}<0.05,{ }^{* *} \mathrm{P}<0.01$; vs $\mathrm{HG}+$ mimics control group, ${ }^{\#} \mathrm{P}<0.05$

with $\mathrm{HG}+$ miR-15b-5p mimics + pcDNA3.1 group. In summary, it revealed that miR-15b-5p affects the HRVEC function through targeting COL12A1.

\section{Circ_001209, a sponge of miR-15b-5p in HRVECs}

CircRNAs, a novel type of non-coding RNAs, have been shown to play an important role in the pathological processes of some diseases through sponging miRNA. We screened circRNAs that might act as a sponge of miR-15b-5p using Targetscan, Starbase, and miRNA.org (Fig. 3A). The predictive results were overlapped with the microarray data, revealing differentially expressed circRNAs between control group and HG-induced HRVECs. Considering abundance and sequence length, the most promising candidates, circ_001209 was screened out through qRT-PCR analysis results (Fig. 3A). Based on the TargetScan prediction (Fig. 3B), we have verified that miR-15b-5p had a binding relationship with circ_001209. And dual luciferase activity results showed that miR-15b-5p mimic could significantly decrease luciferase activity (Fig. 3C). RNA-FISH experiment results showed that circ_001209 and miR-15b-5p were co-localized in the cytoplasm of HRMECs (Fig. 3D). The above results suggest that circ_001209 acts as a sponge of miR-15b-5p in HGinduced HRVECs. 
A

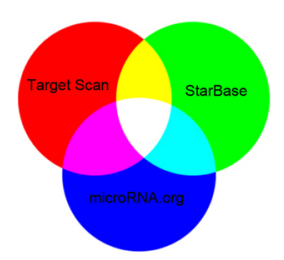

B

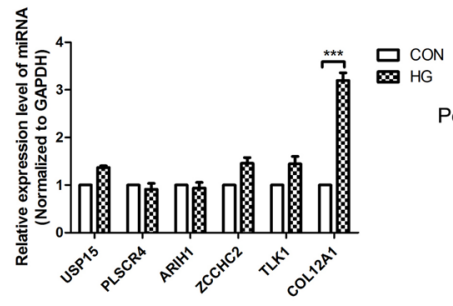

C

osition 2119-2125 of COL12A1 3' UTR 5' ... CUAUGAAGAUAGAAUUGGUGCUU... hsa-miR-15b-5p 3' ACAUUGGUACUAC- ACGACGAU

COL12A1-mut CUAUGAACUUAGAAUAGGUCCAU.

D

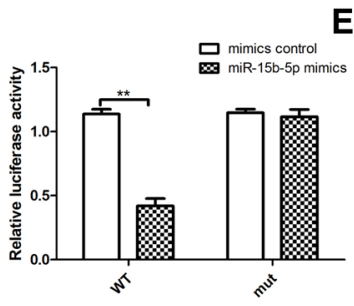

G
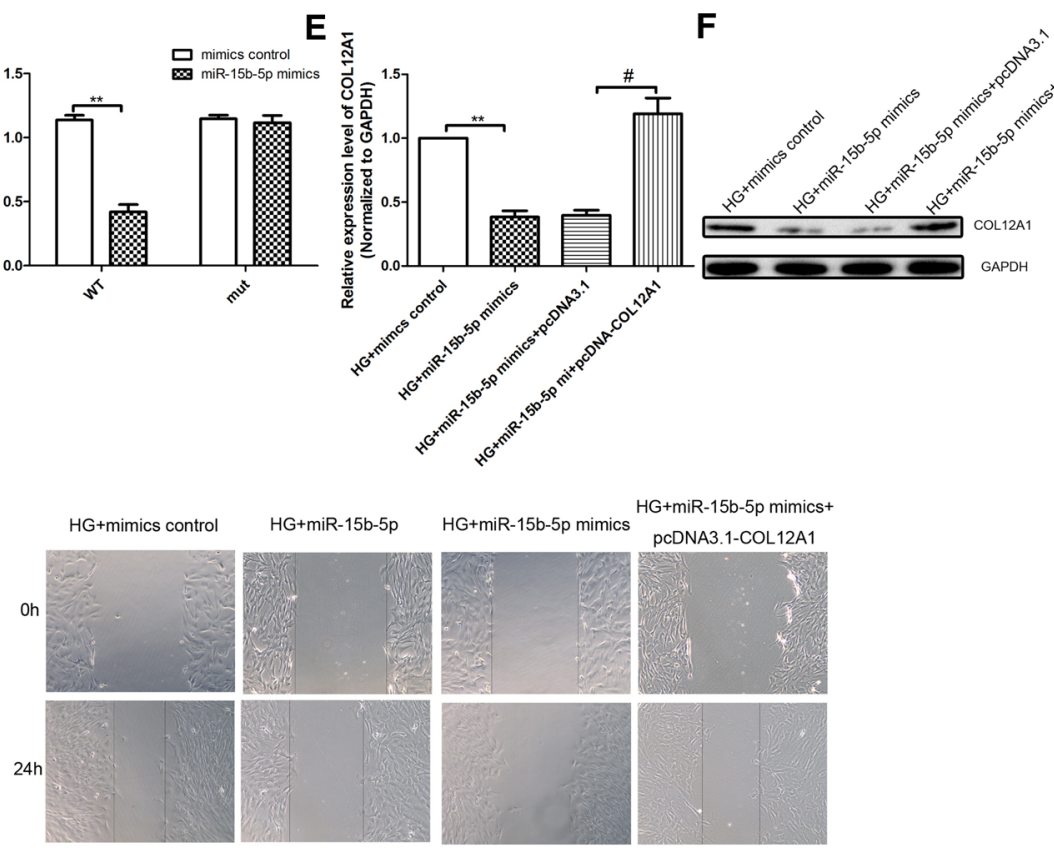

H

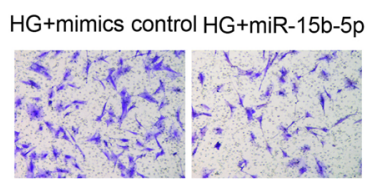

$H G+m i R-15 b-5 p H G+m i R-15 b-5 p$ mimics+ mimics pcDNA3.1-COL12A1
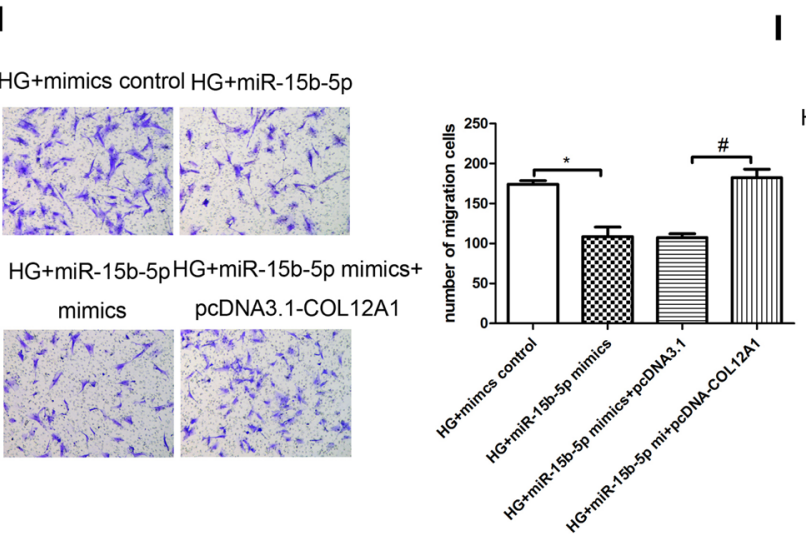

I

$H G+$ mimics control $H G+m i R-15 b-5 p$
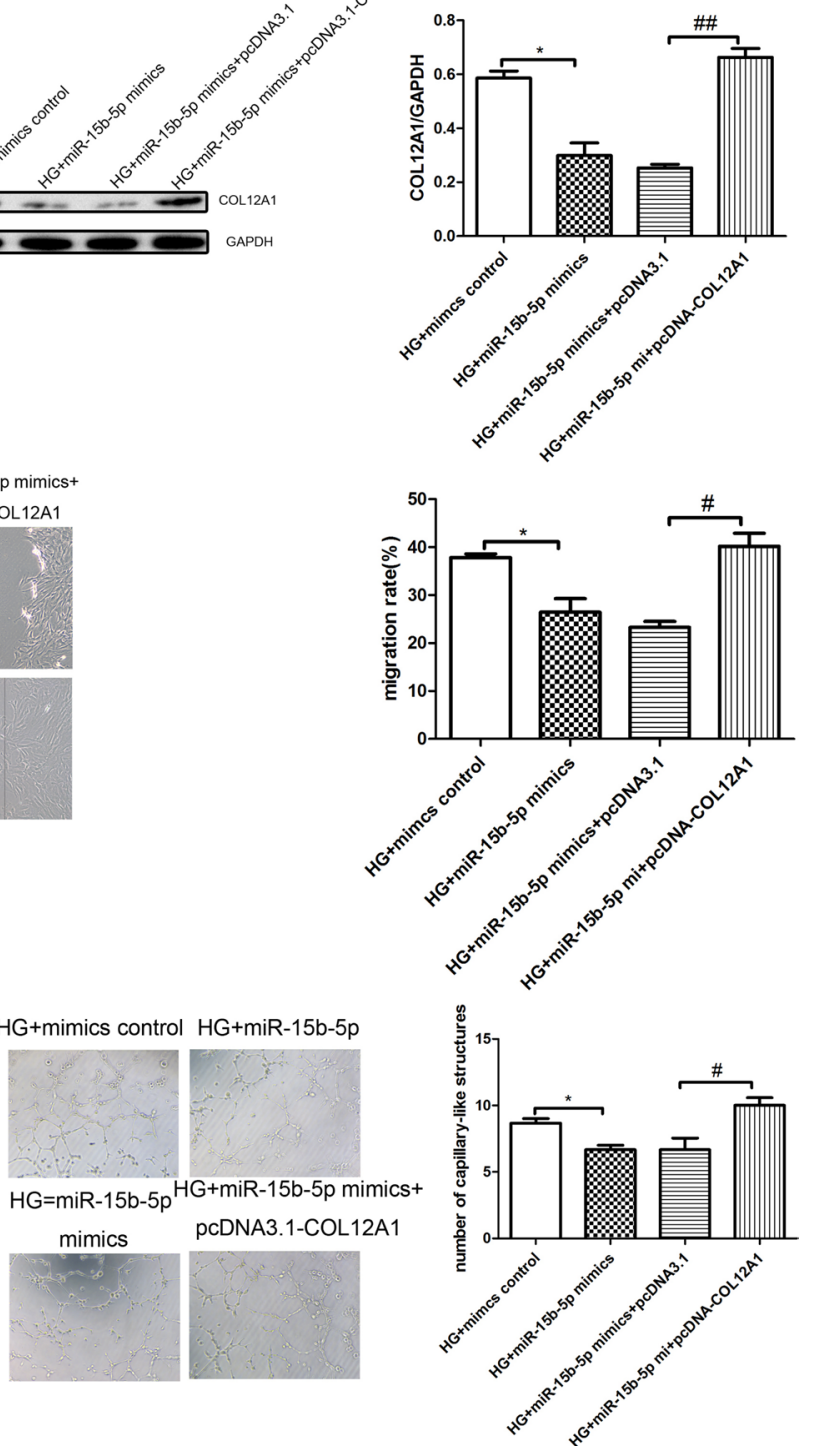

Fig. 2 MiR-15b-5p regulates HRVECs function through targeting COL12A1. A A Veen diagram reveals the predicted target genes of miR-15b-5p from TargetScan, Star Base and microRNA.org. B For the 6 screened target genes, qRT-PCR was used to detect the expression difference induced by HG. C Luciferase reporter vectors containing the wild-type or mutant $3^{\prime}$ UTR of the COL12A1 were constructed according to the predicted binding sites of miR-15b-5p. D The histogram shows the changes of luciferase activities under different conditions. The HRVECs were co-transfected with luciferase reporter vector and miR-15b-5p mimics or mimics control. The expression of COL12A1 in HRVECs induced by HG was detected by qRT-PCR analysis $(\mathbf{E})$ and western blot assay $(\mathbf{F})$. G Wound healing assay was used to detect the invasion of HRVECs in different groups. HTranswell assay was used to detect the migration of HRVECs in different groups. I Tube formation experiment was performed to assess the tubule-forming ability of cells in different groups. The data was presented as mean $\pm \mathrm{SEM}, \mathrm{n}=3$ each group. Vs control/HG + mimics control group, ${ }^{*} \mathrm{P}<0.05$, ${ }^{* *} \mathrm{P}<0.01$; vs HG + miR-15b-5p mimics + pcDNA3.1 group, ${ }^{\#} \mathrm{P}<0.05$, ${ }^{\# \#} \mathrm{P}<0.01$ 


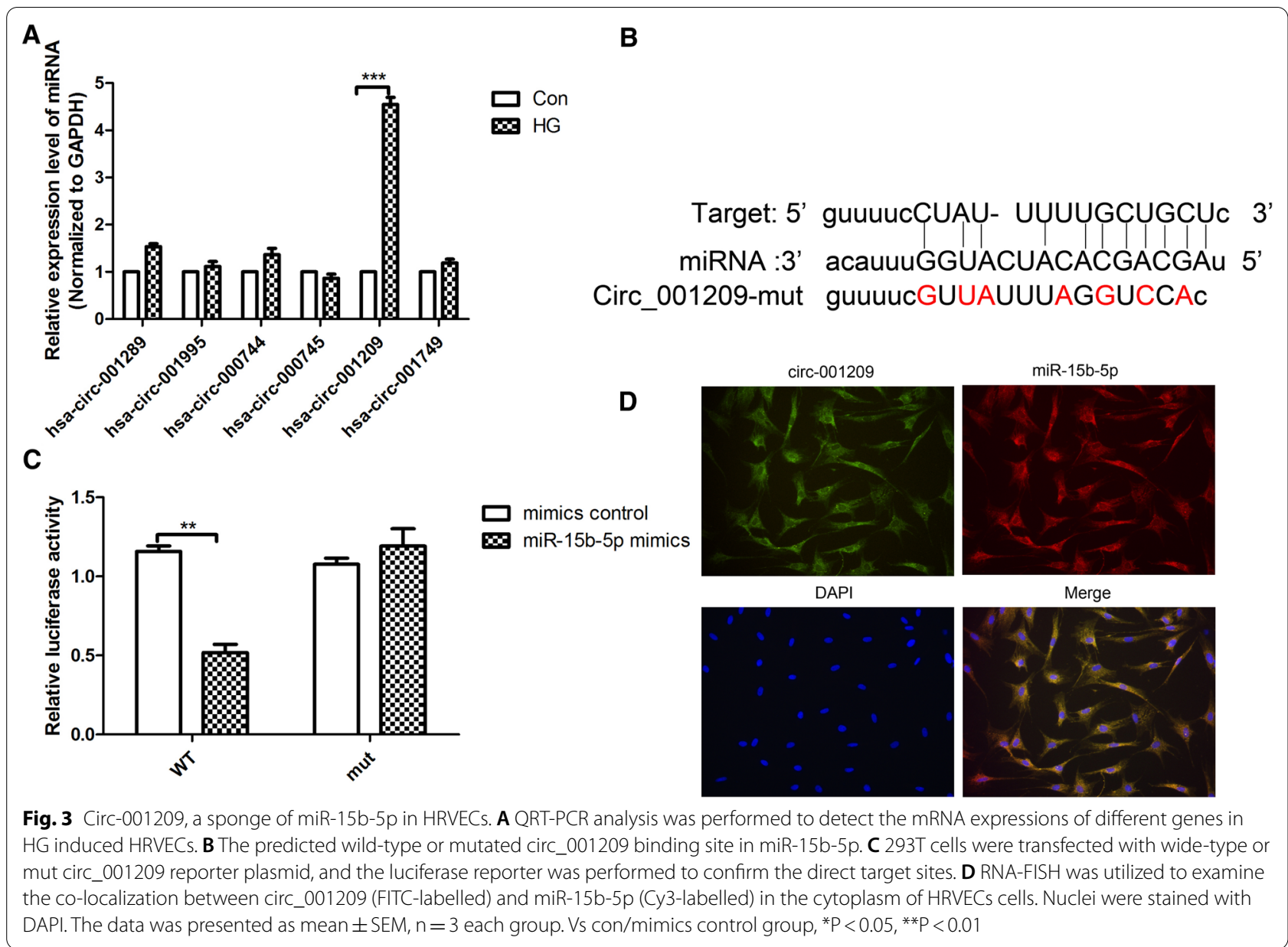

\section{Circ_001209 regulates HRVECs function}

To determine whether circ_001209 regulates HRVEC function by targeting miR-15b-5p, we used lentivirus containing circ_001209 sequence to stabilize the overexpression of circ_001209 in HRVECs. The results (Fig. 4A) showed that compared with ciR-NC group, the mRNA expression of circ_001209 in circ_001209 group was increased significantly. Furthermore, we also detected the changes of miR-15b-5p expression level in HRVECs through qRT-PCR analysis. As shown in Fig. 4B, it revealed that the expression of miR-15b-5p was downregulated significantly in circ_001209 group compared with ciR-NC group. QRT-PCR results (Fig. 4C) showed the mRNA expression of COL12A1 in circ_001209 was increased significantly compared with ciR-NC group. Consistent with qRT-PCR results, western blot results (Fig. 4D) also showed the same trend. Then, the wound healing results, transwell assay results and tube formation results (Fig. 4E-G) showed that compared with con + ciR-NC group, the invasion, migration and tube formation of HRMECs was enhanced in $\mathrm{HG}+$ ciR-NC group significantly. While the invasion, migration and tube formation of HRVECs in HG + circ_001209 group was enhanced significantly compared with HG + ciR-NC group. In summary, it revealed that circ_001209 could affect the HRVEC function.

\section{Circ_001209 regulates HRVEC function through targeting miR-15b-5p}

To address whether circ_001209 regulates HRVEC function through targeting miR-15b-5p, lentivirus containing the circ_001209 sequence was used to stably overexpress circ_001209 in HRVECs. QRT-PCR results (Fig. 5A, B) showed that overexpression of circ_001209 significantly attenuated both the upregulation of miR-15b-5p and the downregulation of COL12A1 in HG induced HRVECs. Besides, western blot results (Fig. 5C) also showed the same trend of COL12A1 in HG-induced HRVECs. Wound healing assay, Transwell assay, and tube formation assays were used to evaluate the roles of circ_001209 under HG conditions in vitro. The results (Fig. 5D-F) showed that compared with $\mathrm{HG}+\mathrm{ciR}-\mathrm{NC}$ group, the 


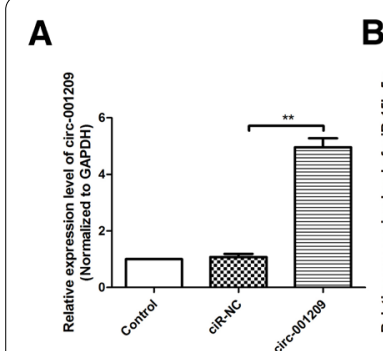

B

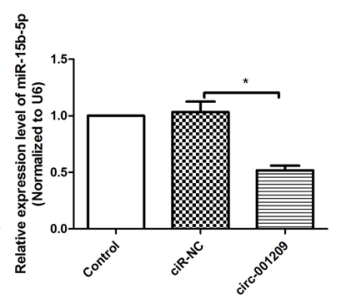

C

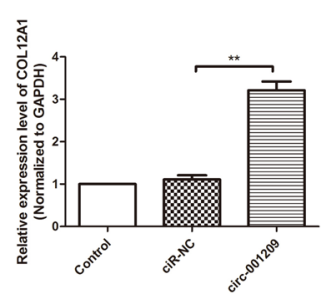

D

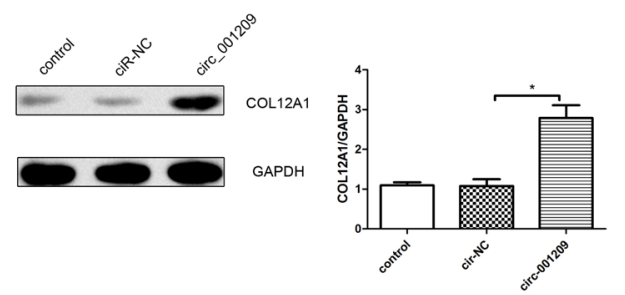

E
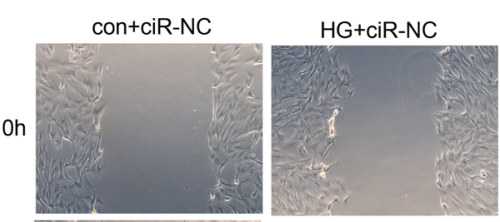

HG+circ-001209
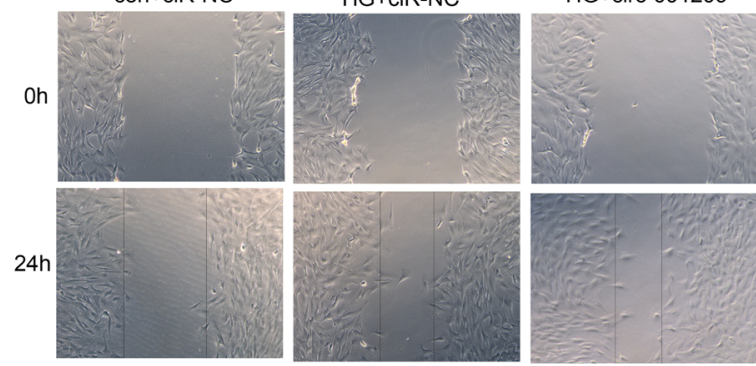

$\mathbf{F}$

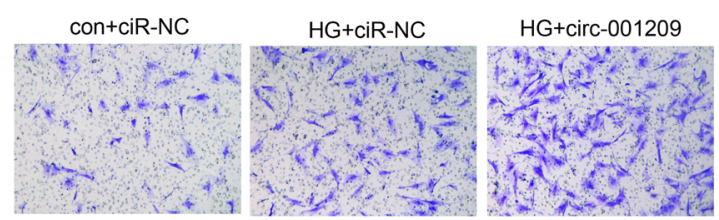

G
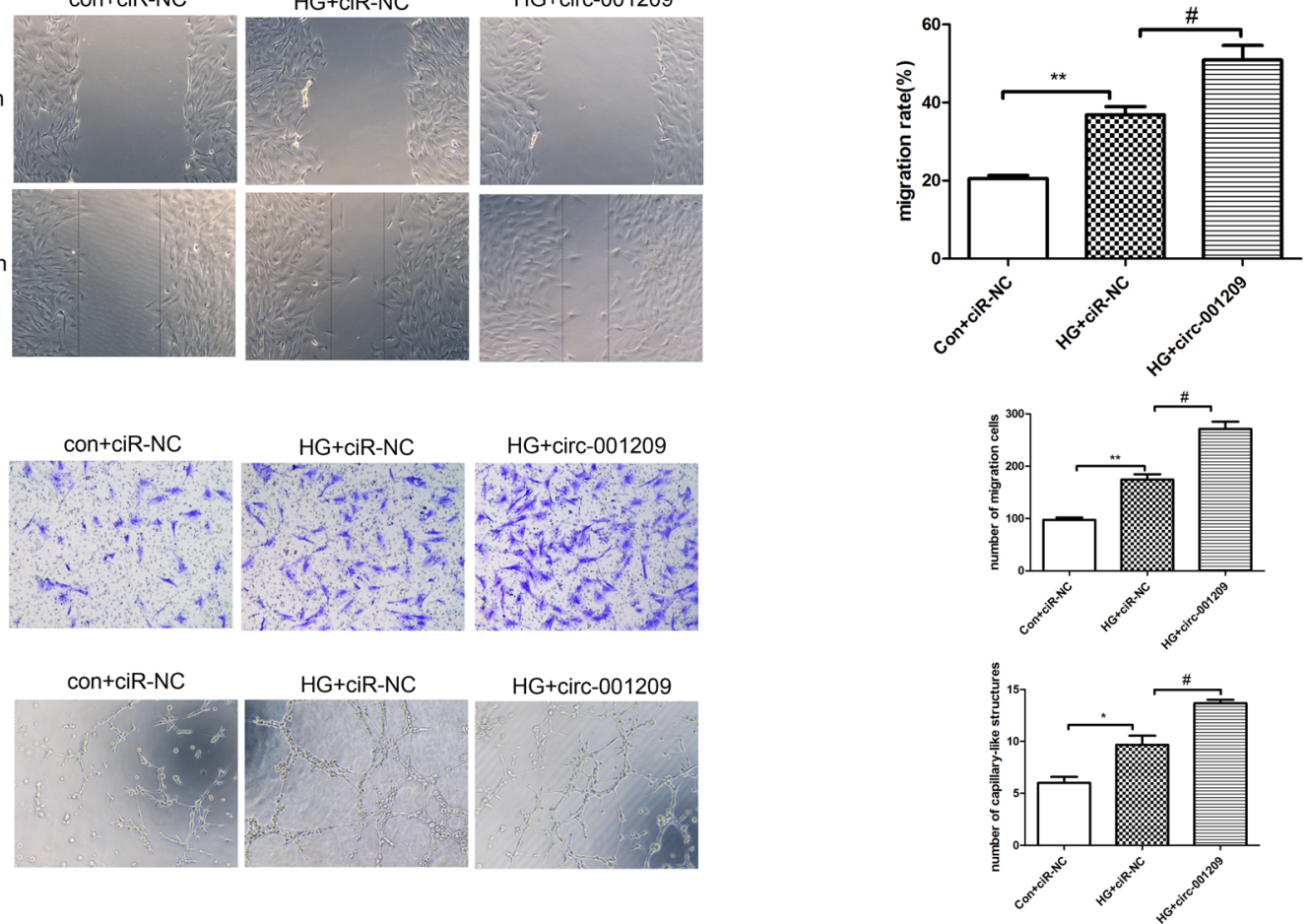

Fig. 4 Circ_001209 regulates HRVECs function. QRT-PCR analysis was used to detect the expressions of circ_001209 (A), miR-15b-5p (B) and COL12A1 $(\mathbf{C})$ in different groups. D Western blot assay was used to detect the protein expression of COL12A1 in different groups. E Wound healing assay was used to detect the invasion of HRVECs in different groups. F Transwell assay was used to detect the migration of HRVECs in different groups. G Tube formation experiment was performed to assess the tubule-forming ability of cells in different groups. The data was presented as mean $\pm S E M, n=3$ each group. Vs con + ciR-NC group, ${ }^{*} P<0.05,{ }^{*} \mathrm{P}<0.01 ; \mathrm{vs} H G+$ ciR-NC group, ${ }^{\#} P<0.05,{ }^{\# \#}<<0.01$

invasion, migration and tube formation of HG induced HRVECs in HG + circ_001209 group was enhanced significantly. Compared with HG + circ_001209+ mimics control group, the invasion, migration and tube formation in HG + circ_001209+ miR-15b-5p mimics group was inhibited significantly. We found that the promoting effects of circ_001209 on invasion, migration, and tube formation in HRVECs were also attenuated by miR-15b-5p mimic transfection under HG conditions. The results suggested that circ_001209 regulates HRVECs function through targeting miR-15b-5p.

\section{Circ_001209 aggravates diabetes mellitus-induced retinal} vascular dysfunction in vivo

We next investigated whether circ_001209 regulates retinal vascular dysfunction in vivo, further affecting visual function in diabetic rats. AAV-circ_001209 was injected intravitreally into diabetic rats. In diabetic retinas injected with AAV-ciR-NC, the level of circ_001209 was significantly increased compared with STZ + AAVciR-NC group. Then, the expression of circ_001209 was strongly increased in AAV-circ_001209-injected diabetic retinas compared with STZ+AAV-ciR-NC 


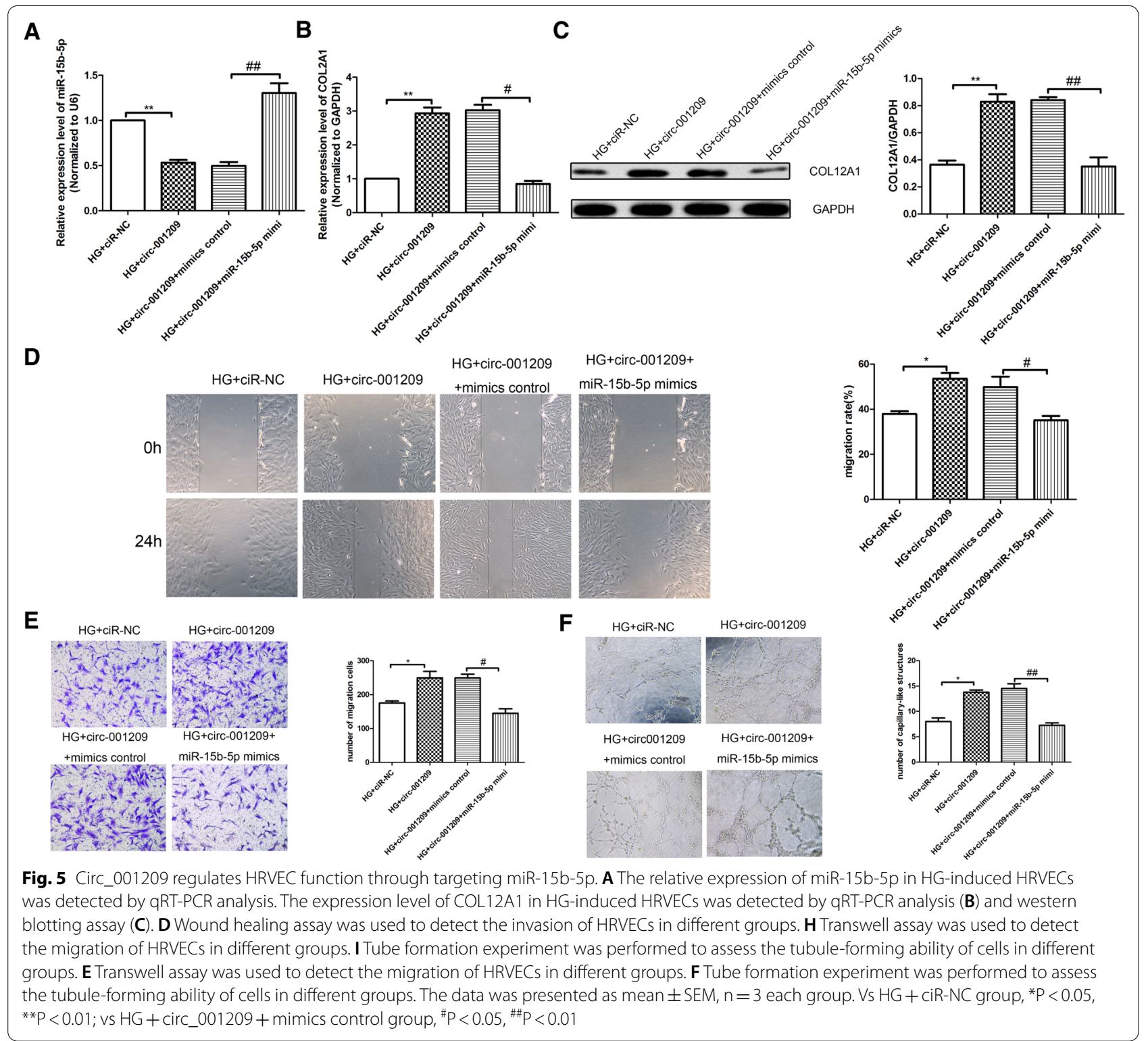

group (Fig. 6A). Similarly, qRT-PCR results (Fig. 6B) showed that the expression of miR-15b-5p in diabetic retinas was reduced significantly compared with sham group. Compared with STZ + AAV-ciR-NC group, the expression of miR-15b-5p was reduced significantly in STZ + AAV-circ_001209 group. We also detected the expression of COL12A1 in diabetic retinas. As shown in Fig. 6C, we found that compared with sham group, the expression of COL12A1 in diabetic retinas of $\mathrm{STZ}+\mathrm{AAV}$-ciR-NC group was significantly increased. The expression of COL12A1 in diabetic retinas of STZ + AAV-circ_001209 group was increased significantly compared with STZ+AAV-ciR-NC group. $H \& E$ staining was used to evaluate the pathological changes of retinopathy, as shown in Fig. 6D. It revealed that compared with sham group, retinal thickness was significantly thinner in diabetic rats. Compared with STZ + AAV-ciR-NC group, the retinal thickness was much thinner in STZ+ AAV-circ_001209 group. TUNEL staining was performed to detect the apoptosis in diabetic retinal, the results (Fig. 6E) showed that the apoptosis in STZ+AAV-ciR-NC group was increased significantly compared with sham group. The apoptosis positive signal in STZ + AAV-circ_001209 group was significantly enhanced compared with STZ + AAVciR-NC group. The results further confirmed that circ_001209 could aggravate diabetes mellitus-induced retinal vascular dysfunction in vivo. 
A

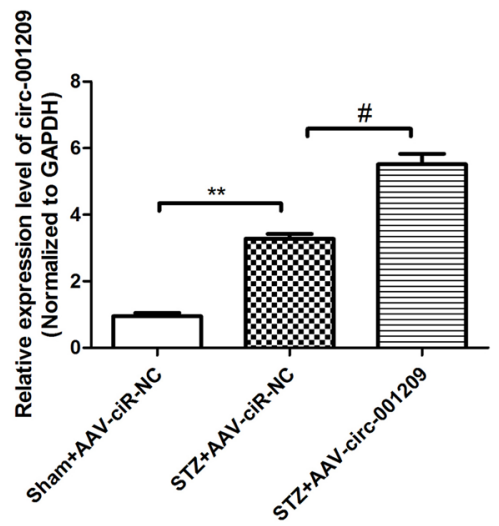

D

sham+AAV-ciR-NC

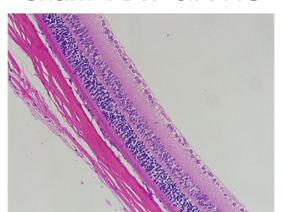

STZ+AAV-ciR-NC

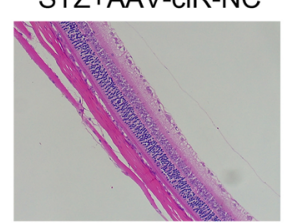

STZ+AAV-circ-001209

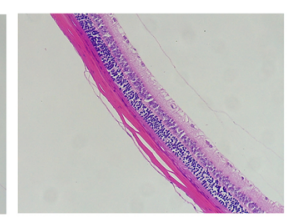

E
B

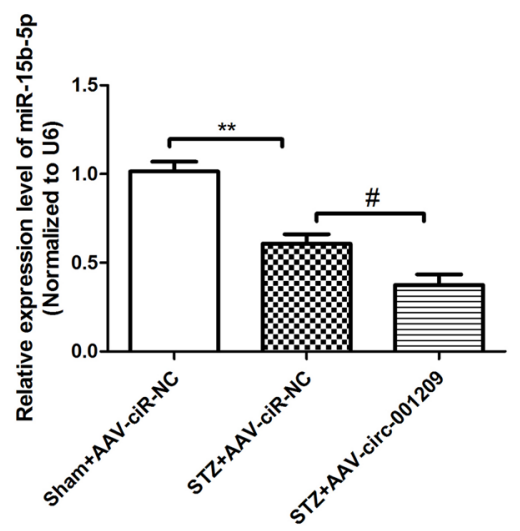

C

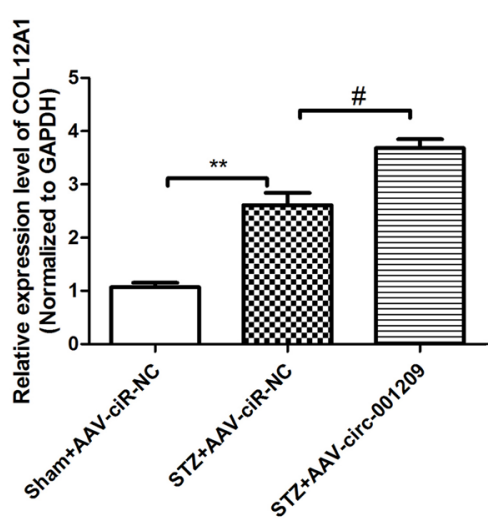

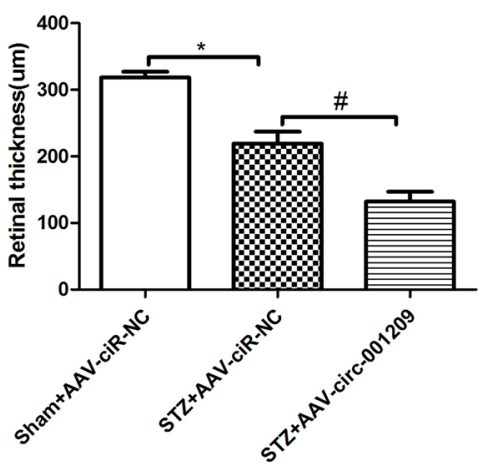
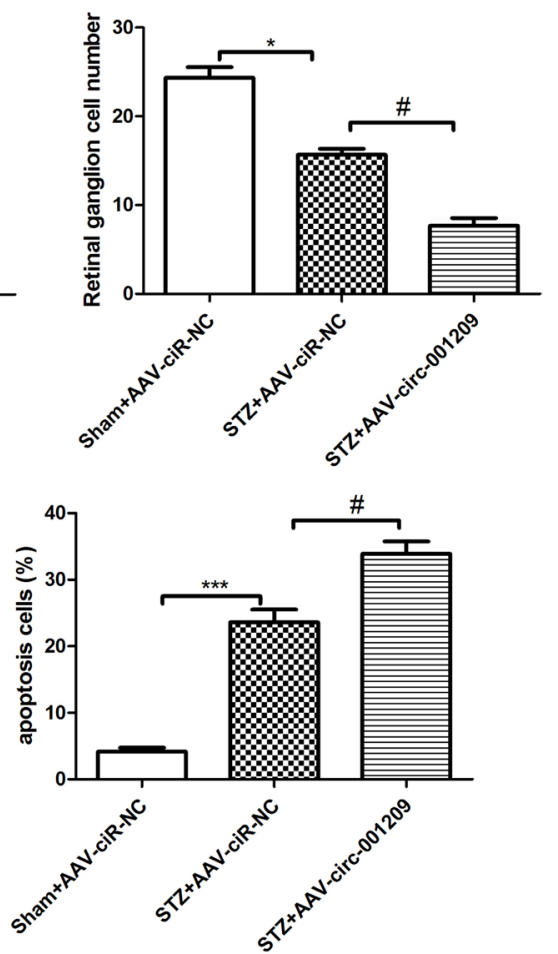

Fig. 6 Circ_001209 aggravates diabetes mellitus-induced retinal vascular dysfunction in vivo. The retinal tissue was taken from the AAV-ciR-NC or AAV-circ_001209 injected diabetic rat at 2 months after injection. The expression of circ_001209 (A), miR-15b-5p (B) and COL12A1 (C) in the retinal tissue was detected by qRT-PCR analysis. Hematoxylin and eosin (H\&E) staining was used to detect the pathological tissue damage induced by diabetic retinopathy in rats. E TUNEL staining images for retinal and the quantization diagram. The data are presented as mean \pm SD. $n=6$ each group. Vs sham \pm AAV-ciR-NC group, ${ }^{* P}<0.05,{ }^{*} P<0.01 ;$ vs STZ + AAV-ciR-NC group, $\# P<0.05$

\section{Discussion}

As a vascular complication in diabetes, DR could lead to blindness in the later stage, seriously affect the quality of life of patients, and also bring economic burden to families and society [20]. Although a large number of studies have explored the pathological mechanism of DR, there is still no effective clinical treatment strategy. Therefore, it is of great clinical significance to clarify the cellular mechanism of DR, develop new treatment strategies and improve the prognosis. The integrity of retinal vascular endothelial cells maintains stable retinal blood flow and ensures the supply of retinal blood oxygen and nutrients [21]. Hyperglycemia induces abnormal activation, proliferation, and migration of retinal vascular endothelial 
cells, which may lead to changes in retinal vascular function. There is increasing evidence [22] that hyperglycemia stimulates retinal vascular endothelial cell dysfunction as the pathological basis of DR.

In addition, previous studies [22] have shown that miRNAs can participate in the regulation of retinal vascular endothelial cell proliferation, activation, apoptosis and other physiological processes in the pathological state of diabetes, thus affecting the function of blood vessels. Zhu et al. [23] found that miR-20b-5p was upregualted markedly in HRVECs and patients with DR, which could promote the proliferation and exacerbate the progression of DR. Whereas, Zeng et al. [24] confirmed that miR29b-3p promoted HRMECs apoptosis, which indicated that miR-29b-3p may be a key factor during the progression of DR. In our study, we found a potential correlation between the downregulation of miR-15b-5p and the dysfunction of retinal vascular endothelial cells. The expression of miR-15b-5p was significantly down-regulated in the HRVECs treated with HG and the retinal tissues of diabetic rats. Besides, the migration and proliferation of human retinal vascular endothelial cells promoted by HG could be significantly inhibited by miR-15b-5p mimics.

COL12A1, a gene encoding collagen type XII $\alpha 1$ chain, is a typical molecule that regulates collagen expression and is involved in the formation of collagen in the cancer microenviroment [25]. Recently, some studies [26] have reported that COL12A1 was associated with the tumor invasion, migration and metastasis, the mechanism in DR has not been reported. In the further study of the regulation mechanism of miR-15b-5p on HRVECs, we found that among the 6 target genes (USP15, PLSCR4, ARIH1, ZCCHC2, TLK1, COL12A1) regulated by miR-15b-5p, the gene level of COL12A1 was the most significantly upregulated in HG induced HRVECs. Besides, cellular functional experiments confirmed that miR-15b-5p directly inhibited the expression of COL12A1 and inhibited the proliferation, migration and angiogenesis of HRVECs induced by HG.

As a new type of non-coding RNA, circRNAs have closed-loop structure and are widely involved in cell proliferation, differentiation, apoptosis and other different cellular physiological or pathological processes [27]. Recent studies [28] have found that abnormal expression of circRNAs may be involved in pathological processes such as inflammation and vascular dysfunction in diabetic retinopathy by sponging microRNAs. Zou et al. [29] suggested that circRNA COL1A2 promoted angiogenesis by regulating the miR-29b/VEGF axis and aggravated the damage of diabetic retinopathy. Qin et al. [30] also found that circRNA-ENF532 plays a protective role in regulating vascular dysfunction in DR. In our study, combined with bioinformatics data analysis, we found that circ_001209 can directly target miR-15b-5p and regulate the expression of COL12A1 in HG induced HRVECs. Consistent with the prediction from bioinformatics, the dual-luciferase reporter assay demonstrated that miR15b-5p binds circ_001209 at the target sites. RNA-FISH analysis showed co-localization of circ_001209 and miR$15 \mathrm{~b}-5 \mathrm{p}$ in the HRVECs. These results demonstrated that circ_001209 functions as a sponge of miR-15b-5p, negatively regulating miR-15b-5p expression under diabetic conditions in retinal vascular tissue.

In the present study, the overexpression of circ_001209 aggravated the abnormal proliferation, migration and tube formation of HRVECs induced by HG. In addition, circ_001209 overexpression significantly increased the tubule-forming ability of retinal blood vessel cells induced by hyperglycemia. In addition, both H\&E and TUNEL staining results in diabetic rats showed that circ_001209 overexpression led to abnormal pathological changes and dysfunction of retinal vessels. As for DR, the exact molecular mechanism of the non-coding RNA regulation still needs to be clarified through future research.

In conclusion, we have provided strong evidence that the upregulation of circ_001209 leads to retinal vascular dysfunction in diabetic retinopathy through the regulation of miR-15b-5p and COL12A1. Since circ_001209 could aggravate the pathological process of diabetic retinopathy, we could explore new therapeutic strategies for diabetic retinopathy in response to this mechanism of action, which will provide new ideas for clinical treatment of diabetic retinopathy.

\section{Acknowledgements}

We are grateful to all participates for their contributions for the present study.

\section{Authors' contributions}

MZ designed the study. FW performed the experiment and collated the data. FW analyzed the data and wrote draft of the manuscript. MZ reviewed and edited the manuscript. All authors read and approved the final version.

\section{Funding}

None.

Availability of data and materials

The data and materials for this study is available from the corresponding author on request if necessary.

\section{Declarations}

Ethics approval and consent to participate

The study protocol was approved by the Ethics Committee of West China Hospital of Sichuan University and performed in strict accordance with the Declaration of Helsinki. Animal experiments were approved by the Animal Ethics Committee and strictly performed according to the Guide for the Care and Use of Laboratory Animals published by the US National Institutes of Health. Extensive efforts were made to reduce animal numbers and suffering.

Consent for publication

Not applicable. 


\section{Competing interests}

The authors declare that they have no competing interests.

\section{Author details}

${ }^{1}$ Department of Ophthalmology, West China Hospital, Sichuan University, Chengdu 610041, Sichuan, China. ${ }^{2}$ Department of Ophthalmology, The Affiliated Hospital of Southwest Medical University, Luzhou, China.

Received: 29 January 2021 Accepted: 18 June 2021

Published online: 07 July 2021

\section{References}

1. Cunha-Vaz J, Conceicao L, et al. Phenotypes and biomarkers of diabetic retinopathy. Progr Retinal Eye Res. 2014;41(1):90-111.

2. Zheng $Y$, He M, Congdon N. The worldwide epidemic of diabetic retinopathy. Indian J Ophthalmol. 2012;60(5):428-31.

3. Lois N, McCarter RV, O'Neill C, Medina RJ, Stitt AW. Endothelial progenitor cells in diabetic retinopathy. Front Endocrinol (Lausanne). 2014;5:44

4. Lee BJ, Byeon HE, Cho CS, et al. Histamine causes an imbalance between pro-angiogenic and anti-angiogenic factors in the retinal pigment epithelium of diabetic retina via $\mathrm{H} 4$ receptor/p38 MAPK axis. BMJ Open Diabetes Res Care. 2020:8:e001710.

5. Min JW, Kim HD, Park SY, et al. Relationship between retinal capillary nonperfusion area and renal function in patients with type 2 diabetes. Invest Ophthalmol Vis Sci. 2020;61:14.

6. Al-Khersan H, Russell JF, Lazzarini TA, et al. Comparison between graders in detection of diabetic neovascularization with swept source OCT angiography and fluorescein angiography. Am J Ophthalmol. 2020:224:292-300.

7. Zhao Q, Zhou F, Zhang Y, et al. Fasting plasma glucose variability levels and risk of adverse outcomes among patients with type 2 diabetes: a systematic review and meta-analysis. Diabetes Res Clin Pract. 2019;148:23-31.

8. Zeng Y, He T, Liu J, et al. Bioinformatics analysis of multi-omics data identifying molecular biomarker candidates and epigenetically regulatory targets associated with retinoblastoma. Medicine (Baltimore). 2020;99:e23314

9. Wang J, Liang S, Guangming W, et al. A novel microRNA-365/SIRT1/ $\beta$ catenin/GLUT1 signaling axis affects the initiation of diabetic retinopathy. Diabetes Metab Res Rev. 2020; e3428.

10. Xue L, Xiong C, Li J, et al. miR-200-3p suppresses cell proliferation and reduces apoptosis in diabetic retinopathy via blocking the TGF- $32 / \mathrm{Smad}$ pathway .Biosci Rep. 2020;40.

11. Ji Q, Han J, Wang $L$, et al. MicroRNA-34a promotes apoptosis of retinal vascular endothelial cells by targeting SIRT1 in rats with diabetic retinopathy. Cell Cycle. 2020;19:2886-96.

12. Ye E-A, Steinle JJ. miR-15b/16 protects primary human retinal microvascular endothelial cells against hyperglycemia-induced increases in tumor necrosis factor alpha and suppressor of cytokine signaling 3. J Neuroinflammation. 2015;12:44.

13. Lasda E, Parker R. Circular RNAs: diversity of form and function. RNA. 2014;20:1829-42.

14. Legnini I, Di Timoteo G, Rossi F, Morlando M, Briganti F, Sthandier O, et al. Circ-ZNF609 is a circular RNA that can be translated and functions in myogenesis. Mol Cell. 2017;66:22-37.
15. Bai Y, Wang J, He Z, Yang M, Li L, Jiang H. Mesenchymal stem cells reverse diabetic nephropathy disease via lipoxin A4 by targeting transforming growth factor beta (TGF-beta)/smad pathway and pro-inflammatory cytokines. Med Sci Monit. 2019;25:3069-76.

16. Zhao L, Li J, Liu Y, et al. Flotillin1 promotes EMT of human small cell lung cancer via TGF- $\beta$ signaling pathway. Cancer Biol Med. 2018;15:400-14.

17. Wang Q, Zhang F, Lei Y, et al. microRNA-322/424 promotes liver fibrosis by regulating angiogenesis through targeting CUL2/HIF-1 a pathway. Life Sci. 2020;266:118819.

18. Gu C, Zhang H, Gao Y. Adipose mesenchymal stem cells-secreted extracellular vesicles containing microRNA-192 delays diabetic retinopathy by targeting ITGA1. J Cell Physiol. 2020;236:5036-51.

19. Chen J, Li X, Yang L, et al. CircASH2L promotes ovarian cancer tumorigenesis, angiogenesis, and lymphangiogenesis by regulating the miR-665/ VEGFA axis as a competing endogenous RNA. Front Cell Dev Biol. 2020;8:595585.

20. Yu L-M, Di W-C, Dong X, et al. Melatonin protects diabetic heart against ischemia-reperfusion injury, role of membrane receptordependent cGMP-PKG activation. Biochim Biophys Acta Mol Basis Dis. 2018;1864:563-78.

21. Klaassen I, Van Noorden CJF, Schlingemann RO. Molecular basis of the inner blood-retinal barrier and its breakdown in diabetic macular edema and other pathological conditions. Prog Retin Eye Res. 2013;34:19-48.

22. Dou X, Duerfeldt AS. Small-molecule modulation of PPARs for the treatment of prevalent vascular retinal diseases. Int J Mol Sci. 2020;21:9251.

23. Zhu K, Hu X, Chen $\mathrm{H}$, et al. Downregulation of circRNA DMNT3B contributes to diabetic retinal vascular dysfunction through targeting miR-20b-5p and BAMBI. EBioMedicine. 2019;49:341-53.

24. Zeng Y, Cui Z, Liu J, et al. MicroRNA-29b-3p promotes human retinal microvascular endothelial cell apoptosis via blocking SIRT1 in diabetic retinopathy. Front Physiol. 2019;10:1621.

25. Karagiannis GS, Berk A, Dimitromanolakis A, et al. Enrichment map profiling of the cancer invasion front suggests regulation of colorectal cancer progression by the bone morphogenetic protein antagonist, gremlin-1. Mol Oncol. 2013;7:826-39.

26. Karagiannis GS, Petraki C, Prassas I, et al. Proteomic signatures of the desmoplastic invasion front reveal collagen type XII as a marker of myofibroblastic differentiation during colorectal cancer metastasis. Oncotarget. 2012;3:267-85

27. Kumar S, Pandey M, Sharawat SK. Biological functions of long noncoding RNAs and circular RNAs in small-cell lung cancer. Epigenomics. 2020;12:1751-63.

28. Wu Z, Liu B, Ma Y, et al. Discovery and validation of hsa_circ_0001953 as a potential biomarker for proliferative diabetic retinopathy in human blood. Acta Ophthalmol. 2020;99:306-13.

29. Zou J, Liu K-C, Wang W-P, et al. Circular RNA COL1A2 promotes angiogenesis via regulating miR-29b/VEGF axis in diabetic retinopathy. Life Sci. 2020;256:117888.

30. Jiang Q, Liu C, Li C-P, et al. Circular RNA-ZNF532 regulates diabetesinduced retinal pericyte degeneration and vascular dysfunction. J Clin Invest. 2020;130:3833-47.

\section{Publisher's Note}

Springer Nature remains neutral with regard to jurisdictional claims in published maps and institutional affiliations. 